\title{
Kyphoscoliotic Ehlers-Danlos syndrome due to FKBP22 deficiency
}

INSERM

\section{Source}

INSERM. (1999). Orphanet: an online rare disease and orphan drug data base.

Kyphoscoliotic Ehlers-Danlos syndrome due to FKBP22 deficiency. ORPHA:300179

Ehlers-Danlos syndrome, kyphoscoliotic and deafness type is a form of Ehlers-Danlos syndrome, characterized by severe generalized hypotonia at birth with severe early-onset kyphoscolosis along with joint hypermobility (without contractures) leading to recurrent dislocations, and sensorineural hearing impairment. 